\title{
Riqueza de formigas (Hymenoptera, Formicidae) epigéicas em povoamentos de Eucalyptus spp. (Myrtaceae) de diferentes idades no Rio Grande do Sul, Brasil
}

\author{
Ricardo Carvalho Fonseca ${ }^{1} \&$ Elena Diehl ${ }^{1,2}$
}

\begin{abstract}
'Programa de Pós-Graduação em Biologia, Universidade do Vale do Rio dos Sinos. Caixa Postal 275, 93001-970 São Leopoldo-RS, Brasil. Endereço eletrônico: ricafo@brturbo.com

${ }^{2}$ Laboratório de Genética de Insetos Sociais, Universidade do Vale do Rio dos Sinos. Caixa Postal 275, 93001-970 São Leopoldo-RS, Brasil. Endereço eletrônico: ediehl@ bios.unisinos.br
\end{abstract}

\begin{abstract}
Richness of epigaeic ants (Hymenoptera, Formicidae) in Eucalyptus spp. plantations with different ages in Rio Grande do Sul, Brazil. The present study describes the epigaeic ant communities in eucalyptus plantations in sandbank in Rio Grande do Sul State. Ant collections were performed in six plantations of Eucalyptus grandis Hill ex Maiden and Eucalyptus saligna Smith with ages varying between five to 31 years old. A total of 24 areas (four/area) were randomly selected for ant collections. In each one, three transects of $100 \mathrm{~m}$ were designed at intervals of $12 \mathrm{~m}$. Along each transect, ten pitfall traps with sardine baits were placed, at intervals of $10 \mathrm{~m}$ for a 24-hour-period. A total of 21,033 ants were collected, belonging to five subfamilies, 12 tribes, 19 genera and 49 species. According to the jackknife estimator of first order, there was no significant difference between the ant communities richness considering the eucalyptus species $(\mathrm{U}=81.500 ;$ g.l. $=1 ; \mathrm{P}=0.582)$ and the ages of the plantations $(\mathrm{U}=2.504 ; \mathrm{g} .1 .=3 ; \mathrm{P}=0.547)$. Results indicate that richness of ant species are not related to the species of eucalyptus and/or to the age of the forests planted in sandbanks.
\end{abstract}

KEYwords. Biodiversity; Eucalyptus; Formicidae; "restingas".

Resumo. Este estudo descreve as comunidades de formigas de solo em povoamentos de eucalipto implantados em ecossistema de restinga no Rio Grande do Sul. As coletas de formigas foram feitas em seis povoamentos de Eucalyptus grandis Hill ex Maiden e de Eucalyptus saligna Smith com idades de 31, 19, sete e cinco anos. Para as coletas de formigas, foram selecionados ao acaso 24 talhões, quatro por povoamento. Em cada talhão, foram traçados três transectos com $100 \mathrm{~m}$ de comprimento, afastados entre si $12 \mathrm{~m}$. Ao longo dos transectos, foram enterradas 30 armadilhas, tipo pitfall, com iscas de sardinha, afastadas entre si $10 \mathrm{~m}$ e mantidas por 24 horas. Foi coletado um total de 21.033 formigas pertencentes a cinco subfamílias, 12 tribos, 19 gêneros e 49 espécies. De acordo com o estimador de riqueza jackknife de primeira ordem, não houve diferenças significativas entre as riquezas das comunidades de formigas considerando as espécies de eucalipto $(\mathrm{U}=81,500 ; \mathrm{g} .1 .=1 ; \mathrm{P}=0,582)$ e as idades dos povoamentos $(\mathrm{U}=2,504 ; \mathrm{g} .1 .=3$; $\mathrm{P}=0,547)$. Os resultados indicam que a riqueza de espécies de formigas não está relacionada à espécie de eucalipto e/ou à idade do povoamento implantado na restinga.

Palavras-chave. Biodiversidade; Eucalyptus; Formicidae; restingas.

A produção e o aproveitamento da madeira são da maior importância, pois existe uma crescente demanda do produto como matéria-prima em vários segmentos produtivos. As plantações comerciais visam suprir essa necessidade, além de reduzir o corte das florestas naturais remanescentes. No entanto, a implantação destas florestas tem como resultado a alteração total dos habitats naturais não só pela modificação do estrato arbóreo, da vegetação arbustiva e herbácea do subbosque (Poggiani \& Oliveira 1998), mas também pela perda da biodiversidade local devido às perturbações causadas pela sua implantação (VIANA 1995). Este fato é um indicador da necessidade de monitoramento da qualidade do solo das mais diversas áreas, sejam elas nativas, florestadas, agropastoris ou urbanas. Para tanto, tem sido sugerido utilizar invertebrados terrestres como bioindicadores (NEw 1996; MCGEOCH \& CHOWN 1998).

Dentre os invertebrados terrestres destacam-se as formigas, encontradas em quase todos os habitats, exceto nos pólos. Em muitas comunidades ecológicas, elas são dominantes, ocupando todos os espaços disponíveis, onde exercem um grande número de funções (SILVA \& BRANDão 1999). Segundo MAJER (1983), apresentam características peculiares tais como: elevada abundância; riquezas de espécies, regional e global, relativamente altas; facilidade de amostragem e de separação em morfo-espécies; vários táxons especializados e sensíveis a alterações do ambiente. Devido a estas características, as formigas têm sido consideradas há algum tempo como candidatas para serem utilizadas como indicadores biológicos do estado de conservação, degradação ou de recuperação dos ecossistemas terrestres (FowLER 1995; MAJER 1996; ANDERSEn 1997; MaJer \& Nichols 1998; Vasconcelos 1998; Lobry De BRUYN 1999).

No Brasil, existem poucos levantamentos taxonômicos da mirmecofauna em áreas de dunas e restingas, dentre os quais pode ser destacado o estudo realizado em Santa Catarina por Bonnet \& Lopes (1993). No caso do Rio Grande do Sul, a 
maioria dos trabalhos realizados está direcionada a grupos específicos de formicídeos (por exemplo, MAYHÉ-NUNES \& Dienl-Fleig 1994; Diehl-Fleig 1997; Gusmão \& LoECK 1999; SANHUdo \& DieHL-Fleig 1999; GrÜRzMacher et al. 2002). Trabalhos referindo-se à diversidade da mirmecofauna neste Estado são incipientes, podendo ser destacados os de HAUBERT et al. (1998); DiEHL-FLeIG et al. (2000); JunQueIRA et al. (2001); SANTOS \& Diehl (2001) e Flores et al. (2002).

No Rio Grande do Sul, as restingas têm sido um dos ecossistemas utilizados para a implantação de florestas comerciais, em especial de eucalipto e pinus, pelo baixo custo de aquisição da terra. A implantação destas florestas altera totalmente o ambiente, surgindo então, uma nova configuração estrutural. Apesar de não se dispor de dados sobre a mirmecofauna em ambientes de restinga livres de ação antrópica, este trabalho objetivou descrever as comunidades de formigas de solo em povoamentos de eucalipto implantados neste ecossistema. A partir dos dados obtidos pretendeu investigar se a riqueza de espécies de formigas estaria relacionada com a espécie de eucalipto e/ou com a idade dos maciços florestais.

\section{MATERIALE MÉTODOS}

O inventário da mirmecofauna foi realizado, de maio a dezembro de 2001, em povoamentos de eucalipto pertencentes à FLOSUL - Florestamento do Sul Ltda., em Capivari do Sul $\left(30^{\circ} 11^{\prime} \mathrm{S} ; 50^{\circ} 21^{\prime} \mathrm{W}\right)$, implantados em restinga.

Os povoamentos de eucalipto da FLOSUL são divididos em talhões de 5,6 ha a 49,8 ha, separados por estradas de terra. Os povoamentos possuem uma altura média de $14 \mathrm{~m}$, cada talhão sendo formado por uma das sete espécies: Eucalyptus citriodora Hook, E. cloeziana F. Muell, E. dunnii Maiden, E. grandis Hill ex Maiden, E. robusta Smith, E. saligna Smith e E. urophylla S.T. Blake, com idades variando de cinco a 31 anos.

As formigas foram coletadas nos povoamentos de $E$. grandis implantados em 1971, 1983 e 1997, portanto, respectivamente, com 31, 19 e cinco anos, e nos povoamentos de E. saligna implantados em 1971, 1995 e 1997, ou seja, com 31, sete e cinco anos, respectivamente. Todos os talhões avaliados caracterizam-se pela ausência de sub-bosque, uma vez que, até 1993, os plantios sofriam capinas manuais e, a partir de 1994, roçadas e capinas químicas na linha de plantio.

A partir do mapa da propriedade a ser amostrada, foram selecionados aleatoriamente 24 talhões. Para a coleta das formigas, em cada talhão foram traçados três transectos de $100 \mathrm{~m}$, com espaçamento de $12 \mathrm{~m}$ entre eles. Ao longo de cada transecto foram colocadas dez armadilhas tipo "pitfall" com iscas de sardinha, a intervalos de $10 \mathrm{~m}$, totalizando 30 armadilhas por talhão.

As armadilhas utilizadas para a coleta são compostas de um copo plástico de $300 \mathrm{~mL}$, enterrado até a borda superior, contendo $50 \mathrm{~mL}$ de álcool $70 \%$, uma haste de arame galvanizado contendo na porção superior papel alumínio com uma isca de sardinha homogeneizada em óleo vegetal comestível.
As armadilhas foram recolhidas após 24 horas e o material coletado foi acondicionado em vidros com álcool 70\%, devidamente etiquetados, para posterior triagem e identificação. Em laboratório, as formigas foram identificadas ao nível de gênero, segundo a chave dicotômica de BoLToN (1994). Foram feitas separações em morfo-espécies de acordo com os padrões da Coleção de Referência de Formicidae do Laboratório de Genética de Insetos Sociais da Universidade do Vale do Rio dos Sinos (UNISINOS). Quando possível, a identificação foi feita em nível de espécie por comparação com os exemplares existentes na mesma Coleção, na qual os espécimes testemunho foram depositados.

Os dados de abundância relativa foram calculados com base na frequiência de ocorrência das espécies nas iscas e não com base no número de indivíduos. Tal procedimento é mais apropriado para comparações interespecíficas, pois os cálculos não sofrem os efeitos do tamanho das colônias, nem do comportamento de recrutamento das operárias (ROMERo \& JAFFÉ 1989), o que levaria a superestimar aquelas espécies com sistemas de recrutamento mais eficientes e/ou aquelas cujas colônias estão mais próximas das iscas (LEAL \& LoPEs 1992; TAVARES et al. 2001).

Por espécie de eucalipto e idade do povoamento, a riqueza observada foi calculada pelo somatório das ocorrências das espécies de formigas coletadas no conjunto de armadilhas de cada talhão. A projeção da riqueza foi obtida por meio do estimador de riqueza jackknife de primeira ordem, calculado através do programa EstimateS, versão 5.0.1 (CoLwell 1997). Para detectar possíveis diferenças entre as idades dos povoamentos e entre as espécies de eucalipto, foi feita análise não-paramétrica de Kruskal-Wallis.

\section{RESULTADOS EDISCUSSÃO}

Nos seis povoamentos de eucalipto implantados em ecossistema de restinga, foram coletadas 21.033 formigas, tendo sido identificadas 49 espécies, representantes de 19 gêneros, 12 tribos e cinco subfamílias (Tabela I). Este resultado é semelhante aos números obtidos por TAVAREs et al. (2001) que encontraram 45 espécies de formigas distribuídas em 16 gêneros pertencentes a cinco subfamílias em povoamentos de E. cloeziana F. Muell em região de cerrado. Os autores avaliaram as comunidades de formigas no centro e na borda dos talhões de eucalipto, e em vegetação de cerrado adjacente, sugerindo que a baixa riqueza encontrada estaria relacionada à homogeneidade estrutural do eucaliptal e à ausência do subbosque. A reduzida riqueza de espécies encontrada nos povoamentos implantados em restinga possivelmente também está associada à baixa complexidade estrutural da vegetação local, expressa pela ausência de sub-bosque. Comparando-se as restingas do sul do Brasil, localizadas em região sub-tropical, com as áreas de cerrado, em região tropical, seria esperada uma maior riqueza de espécies de formigas nos cerrados. A ausência de diferenças entre as duas áreas pode decorrer de diferentes esforços amostrais ou estar refletindo uma característica própria de florestas de eucalipto. Sabe-se que, 
Tabela I. Espécies de formigas amostradas no solo em povoamentos de eucalipto com diferentes idades implantados em ecossistemas de restinga, Capivari do Sul, RS. Maio a dezembro de 2001.

\begin{tabular}{|c|c|c|c|c|c|c|c|c|}
\hline \multirow{3}{*}{ Subfamílias } & \multirow{3}{*}{ Tribos } & \multirow{3}{*}{ Espécies } & \multicolumn{6}{|c|}{ Idade (anos) } \\
\hline & & & \multicolumn{2}{|c|}{31} & \multirow{2}{*}{$\frac{19}{E}$} & \multirow{2}{*}{$\frac{7}{E .}$} & \multicolumn{2}{|l|}{5} \\
\hline & & & $\begin{array}{c}E . \\
\text { saligna }\end{array}$ & $\begin{array}{c}E . \\
\text { grandis }\end{array}$ & & & $\begin{array}{c}E . \\
\text { saligna }\end{array}$ & $\begin{array}{c}E . \\
\text { grandis }\end{array}$ \\
\hline \multirow[t]{3}{*}{ Dolichoderinae } & Dolichoderini & Dorymyrmex sp. & $\mathrm{X}$ & $\mathrm{X}$ & - & $\mathrm{X}$ & $\mathrm{X}$ & $\mathrm{X}$ \\
\hline & & Dorymyrmex sp.1 & - & $\mathrm{x}$ & - & - & - & - \\
\hline & & Linepithema sp. & $\mathrm{x}$ & $\mathrm{x}$ & $\mathrm{x}$ & $\mathrm{x}$ & $\mathrm{x}$ & $\mathrm{x}$ \\
\hline \multirow[t]{11}{*}{ Formicinae } & Camponotini & Camponotus rufipes (Fabricius) & - & $\mathrm{x}$ & $\mathrm{x}$ & $\mathrm{x}$ & $\mathrm{x}$ & $\mathrm{x}$ \\
\hline & & Camponotus fastigatus Roger & - & $\mathrm{x}$ & - & - & $\mathrm{x}$ & - \\
\hline & & Camponotus mus Roger & $\mathrm{x}$ & $\mathrm{x}$ & $\mathrm{X}$ & $\mathrm{x}$ & $\mathrm{x}$ & $\mathrm{x}$ \\
\hline & & Camponotus sp.1 & $\mathrm{x}$ & $\mathrm{x}$ & $\mathrm{x}$ & $\mathrm{x}$ & $\mathrm{x}$ & $\mathrm{x}$ \\
\hline & & Camponotus sp.6 & $\mathrm{x}$ & $\mathrm{x}$ & $\mathrm{x}$ & $\mathrm{x}$ & $\mathrm{x}$ & $\mathrm{x}$ \\
\hline & & Camponotus sp.11 & $\mathrm{x}$ & $\mathrm{x}$ & - & - & - & - \\
\hline & & Camponotus sp.13 & - & - & - & - & - & $\mathrm{x}$ \\
\hline & & Camponotus sp.14 & - & - & - & - & $\mathrm{x}$ & - \\
\hline & & Camponotus sp.15 & - & $\mathrm{x}$ & $\mathrm{x}$ & $\mathrm{x}$ & $\mathrm{x}$ & - \\
\hline & Lasiini & Paratrechina sp. & $\mathrm{x}$ & $\mathrm{x}$ & - & $\mathrm{x}$ & $\mathrm{x}$ & $\mathrm{x}$ \\
\hline & & Paratrechina sp. 2 & - & - & - & $\mathrm{x}$ & $\mathrm{x}$ & - \\
\hline \multirow[t]{13}{*}{ Myrmicinae } & Attini & Acromyrmex ambiguus Emery & - & - & - & $\mathrm{x}$ & $\mathrm{x}$ & - \\
\hline & & Acromyrmex laticeps Emery & - & $\mathrm{x}$ & $\mathrm{x}$ & $\mathrm{x}$ & $\mathrm{x}$ & $\mathrm{x}$ \\
\hline & & Acromyrmex lundi (Guérin) & - & $\mathrm{x}$ & $\mathrm{x}$ & - & $\mathrm{x}$ & $\mathrm{x}$ \\
\hline & & Apterostigma sp. & $\mathrm{x}$ & - & $\mathrm{x}$ & - & $\mathrm{x}$ & - \\
\hline & & Cyphomyrmex sp.1 & $\mathrm{x}$ & $\mathrm{x}$ & - & $\mathrm{x}$ & $\mathrm{x}$ & $\mathrm{x}$ \\
\hline & & Trachymyrmex sp.1 & $\mathrm{x}$ & - & - & - & $\mathrm{x}$ & - \\
\hline & Crematogastrini & Crematogaster quadriformis Roger & $\mathrm{x}$ & $\mathrm{x}$ & $\mathrm{x}$ & - & - & - \\
\hline & & Crematogaster sp. prox. quadriformis & is $\mathrm{x}$ & $\mathrm{x}$ & $\mathrm{x}$ & $\mathrm{x}$ & $\mathrm{x}$ & $\mathrm{x}$ \\
\hline & Myrmicini & Hylomyrma sp.1 & $\mathrm{x}$ & $\mathrm{x}$ & - & $\mathrm{x}$ & $\mathrm{x}$ & $\mathrm{x}$ \\
\hline & Pheidolini & Pheidole fimbriata Roger & $\mathrm{x}$ & $\mathrm{x}$ & $\mathrm{x}$ & $\mathrm{x}$ & $\mathrm{x}$ & $\mathrm{x}$ \\
\hline & & Pheidole diligens (Fr. Smith) & - & - & - & $\mathrm{x}$ & - & - \\
\hline & & Pheidole triconstricta Forel & $\mathrm{x}$ & $\mathrm{x}$ & $\mathrm{x}$ & - & $\mathrm{x}$ & - \\
\hline & & Pheidole sp.2 & $\mathrm{x}$ & - & - & $\mathrm{x}$ & $\mathrm{x}$ & - \\
\hline \multirow[t]{13}{*}{ Myrmicinae } & Pheidolini & Pheidole sp.3 & $\mathrm{x}$ & $\mathrm{x}$ & - & $\mathrm{x}$ & - & - \\
\hline & & Pheidole sp.5 & $\mathrm{x}$ & $\mathrm{x}$ & - & $\mathrm{x}$ & - & $\mathrm{x}$ \\
\hline & & Pheidole sp.6 & $\mathrm{x}$ & $\mathrm{x}$ & $\mathrm{x}$ & $\mathrm{x}$ & $\mathrm{x}$ & $\mathrm{x}$ \\
\hline & & Pheidole sp.7 & - & $\mathrm{x}$ & - & - & - & $\mathrm{x}$ \\
\hline & & Pheidole sp.10 & $\mathrm{x}$ & $\mathrm{x}$ & $\mathrm{x}$ & - & $\mathrm{x}$ & $\mathrm{x}$ \\
\hline & & Pheidole sp.11 & $\mathrm{x}$ & $\mathrm{x}$ & $\mathrm{x}$ & $\mathrm{x}$ & $\mathrm{x}$ & $\mathrm{x}$ \\
\hline & & Pheidole sp.13 & - & $\mathrm{x}$ & - & - & $\mathrm{x}$ & $\mathrm{x}$ \\
\hline & & Pheidole sp.15 & $\mathrm{x}$ & - & - & $\mathrm{x}$ & $\mathrm{x}$ & $\mathrm{x}$ \\
\hline & & Pheidole sp.18 & $\mathrm{x}$ & $\mathrm{x}$ & - & - & - & - \\
\hline & & Pheidole sp.21 & - & $\mathrm{x}$ & - & - & - & - \\
\hline & Blepharidattini & Wasmannia sp.2 & $\mathrm{x}$ & $\mathrm{x}$ & $\mathrm{x}$ & $\mathrm{x}$ & $\mathrm{x}$ & $\mathrm{x}$ \\
\hline & Solenopsidini & Solenopsis invicta Buren & - & $\mathrm{x}$ & $\mathrm{x}$ & $\mathrm{x}$ & $\mathrm{x}$ & $\mathrm{x}$ \\
\hline & & Solenopsis sp.2 & $\mathrm{x}$ & $\mathrm{x}$ & $\mathrm{x}$ & $\mathrm{x}$ & $\mathrm{x}$ & $\mathrm{x}$ \\
\hline \multirow[t]{8}{*}{ Ponerinae } & Ectatommini & Ectatomma edentatum Roger & - & - & $\mathrm{x}$ & - & - & - \\
\hline & Ponerini & Anochetus sp. & - & - & - & - & $\mathrm{x}$ & - \\
\hline & & Hypoponera opaciceps (Mayr) & $\mathrm{x}$ & - & $\mathrm{x}$ & - & $\mathrm{x}$ & $\mathrm{x}$ \\
\hline & & Hypoponera sp. & $\mathrm{x}$ & $\mathrm{x}$ & $\mathrm{X}$ & $\mathrm{x}$ & $\mathrm{x}$ & - \\
\hline & & Pachycondyla striata Fr. Smith & - & - & $\mathrm{x}$ & - & - & - \\
\hline & & Pachycondyla sp. & - & - & $\mathrm{X}$ & - & - & - \\
\hline & & Odontomachus chelifer (Latreille) & - & - & $\mathrm{x}$ & $\mathrm{x}$ & $\mathrm{x}$ & $\mathrm{x}$ \\
\hline & & Odontomachus sp.1 & - & - & - & - & $\mathrm{x}$ & $\mathrm{x}$ \\
\hline $\begin{array}{l}\text { Pseudomyr- } \\
\text { mecinae }\end{array}$ & Pseudomyrmecini & Pseudomyrmex sp.4 & - & $\mathrm{X}$ & $\mathrm{X}$ & $\mathrm{X}$ & - & - \\
\hline 5 & 2 & 49 & 27 & 33 & 26 & 28 & 35 & 27 \\
\hline
\end{tabular}


quanto maior a complexidade ambiental, maior a sua biodiversidade e menor a abundância de cada espécie. No caso específico da região tropical, a riqueza de espécies de formigas é menor em ambientes homogêneos com baixa complexidade estrutural da vegetação do que nos ambientes heterogêneos com alta complexidade vegetacional (MATOs et al. 1994).

Nas plantações de eucalipto avaliadas neste trabalho, a subfamília Myrmicinae apresentou a maior riqueza de espécies $(\mathrm{S}=26)$, tendo sido encontrado para o gênero Pheidole Westwood 1841 a maior riqueza específica (14 espécies). A mesma situação tem sido verificada em vários levantamentos da mirmecofauna nos mais diversos habitats (LOPES \& LEAL 1991; CAldas \& Moutinho 1993; TAVARES et al. 2001; Marinho et al. 2002). Na região Neotropical, podem ser listados mais de 400 nomes representados por Pheidole (KeMPF 1972), ainda que, em levantamentos de mirmecofauna raramente sejam relatados mais do que 20 espécies em uma determinada localidade.

A subfamília Formicinae apresentou a segunda maior riqueza específica, tendo o gênero Camponotus Mayr 1861 o maior número de espécies (9). Resultado similar foi encontrado por MARINHO et al. (2002) que associaram a elevada riqueza deste gênero ao fato dele possuir um dos maiores números de espécies descritas em nível mundial (HöLLDOBLER \& WiLSON 1990; Bolton 1995), a melhor distribuição geográfica e apresentar a maior diversidade de adaptações ecológicas (WILSON 1976).

Das 49 espécies de formigas encontradas, apenas dez $(20,41 \%)$ foram comuns aos seis povoamentos de eucalipto. Paralelamente, Dorymyrmex sp. 1 e Pheidole sp. 21 foram exclusivas dos povoamentos de E. grandis com 31 anos de idade; Ectatomma edentatum Roger 1863, Pachycondyla striata Fr. Smith 1858 e Pachycondyla sp. exclusivas das parcelas com 19 anos; Pheidole diligens (Fr. Smith 1858) dos com sete anos; Anochetus sp., Camponotus sp. 14 (E. saligna) e Camponotus sp. 13 (E. grandis) exclusivas dos povoamentos com cinco anos de idade (Tabela I).

Nos seis povoamentos (Tabela II), as sete espécies com maior frequiência média de ocorrência foram: Pheidole sp. 11 (100\%), Pheidole fimbriata Roger 1863 (95,8\%), Pheidole sp.
$6(91,7 \%)$, Solenopsis sp. 2 (83,3\%), Wasmannia sp. $2(70,8 \%)$ Crematogaster sp. prox. quadriformis $(66,7 \%)$ e Acromyrmex laticeps Emery 1905 (62,5\%).

Considerando as duas espécies de eucalipto e de acordo com o estimador de riqueza jackknife de primeira ordem, a comparação das comunidades de formigas não apresentou diferença significativa $(\mathrm{U}=81,500 ; \mathrm{g} .1 . \mathrm{l}=1 ; \mathrm{P}=0,582)$. Mas, embora não significativa, a riqueza de espécies de formigas estimada $\left(\mathrm{S}_{\text {est }}=47\right)$ para o povoamento com E. saligna implantado em 1997 foi bem superior em relação aos outros anos de implantação para a mesma espécie de eucalipto, bem como quando comparado com E. grandis (Tabela III).

Da mesma forma que para as espécies de eucalipto, ao se comparar as riquezas estimadas $\left(\mathrm{S}_{\text {est }}\right)$ para as idades dos povoamentos (Tabela III), não foram encontradas diferenças significativas $(\mathrm{U}=2,504 ; \mathrm{g} .1 .=3 ; \mathrm{P}=0,547)$ apesar de ter ocorrido uma pequena variação na composição de espécies entre as comunidades de formigas (Tabela I). A ausência de diferenças significativas entre as comunidades de formigas nos povoamentos de eucalipto com diferentes idades pode estar relacionada à falta de sub-bosque, pois segundo OLIVEIRA et al. (1995) em ambientes homogêneos e estruturalmente simplificados, como os de eucaliptais, não há uma variação significativa da riqueza de espécies de formigas. No presente estudo, a riqueza média de formigas não diferiu estatisticamente entre os diversos talhões avaliados indicando, portanto, a ausência de relação entre a espécie de eucalipto e/ou a idade do povoamento e a riqueza de espécies de formigas.

Três espécies do gênero Pheidole e uma de Solenopsis Westwood 1841 merecem especial atenção pela alta freqüência de ocorrência nos talhões de eucalipto (Tabela II). Estes dois gêneros estão entre os que apresentam alta riqueza de espécies em regiões tropicais (WILSON 1976) com tendência a serem predominantes em todos ecossistemas terrestres uma vez que as suas espécies possuem ampla tolerância às condições físicas do ambiente (ANDERSEN 1991). A elevada freqüência das espécies destes dois gêneros, em relação a outras espécies de formigas, poderia estar relacionada com um recrutamento em massa mais eficiente (Caldas \& Moutinho 1993). Adicionalmente, espécies de Solenopsis estão entre as mais agressivas na utilização de recursos (MARINHO et al. 2002),

Tabela II. Freqüências relativas médias de ocorrência, por talhão, de espécies de formigas de solo em seis povoamentos de eucalipto em ecossistema de restinga, Capivari do Sul, RS. Maio a dezembro de 2001.

\begin{tabular}{|c|c|c|c|c|c|c|}
\hline \multirow{3}{*}{ Espécies } & \multicolumn{6}{|c|}{ Idade (anos) } \\
\hline & \multicolumn{2}{|c|}{31} & \multirow{2}{*}{$\begin{array}{c}19 \\
\text { E. saligna }\end{array}$} & \multirow{2}{*}{$\frac{7}{\text { E. grandis }}$} & \multicolumn{2}{|c|}{5} \\
\hline & E. saligna & E. grandis & & & E. saligna & E. grandis \\
\hline Pheidole sp.11 & 100,0 & 100,0 & 100,0 & 100,0 & 100,0 & 100,0 \\
\hline Pheidole fimbriata Roger & 75,0 & 100,0 & 100,0 & 100,0 & 100,0 & 100,0 \\
\hline Pheidole sp.6 & 75,0 & 100,0 & 100,0 & 75,0 & 100,0 & 100,0 \\
\hline Solenopsis sp. 2 & 75,0 & 100,0 & 75,0 & 75,0 & 100,0 & 100,0 \\
\hline Wasmannia sp.2 & 50,0 & 75,0 & 25,0 & 100,0 & 100,0 & 75,0 \\
\hline Crematogaster sp. prox. quadriformis & 75,0 & 100,0 & 25,0 & 50,0 & 100,0 & 50,0 \\
\hline Acromyrmex laticeps Emery & - & 75,0 & 100,0 & 75,0 & 75,0 & 25,0 \\
\hline
\end{tabular}


Tabela III. Riqueza média de espécies por talhão $\left(\mathrm{S}_{\mathrm{M}}\right)$, riqueza observada $\left(\mathrm{S}_{\mathrm{obs}}\right)$ e estimativa de riqueza $\left(\mathrm{S}_{\mathrm{est}}\right)$ de espécies de formigas de solo em seis povoamentos de eucalipto implantados em ecossistema de restinga, Capivari do Sul, RS. Maio a dezembro de 2001.

\begin{tabular}{|c|c|c|c|c|c|}
\hline $\begin{array}{c}\text { Ano de } \\
\text { implantação }\end{array}$ & Idade & Eucalipto & $\mathrm{S}_{\mathrm{M}}$ & $\mathrm{S}_{\mathrm{obs}}$ & $\mathrm{S}_{\text {est }}$ \\
\hline \multirow[t]{2}{*}{1971} & 31 & E. saligna & 13,00 & 27 & 35 \\
\hline & & E. grandis & 18,75 & 33 & 37 \\
\hline 1983 & 19 & E. saligna & 13,50 & 26 & 36 \\
\hline 1995 & 7 & E. grandis & 13,50 & 28 & 38 \\
\hline \multirow[t]{2}{*}{1997} & 5 & E. saligna & 18,25 & 35 & 47 \\
\hline & & E. grandis & 15,00 & 27 & 34 \\
\hline
\end{tabular}

podendo passar longos períodos de escassez de alimento e competir por estes com outras espécies, seja de formigas ou de outros grupos animais (Fowler et al. 1991). As altas freqüências de ocorrência nas áreas amostradas associadas a alterações da estrutura do ambiente, devido ao manejo a que são submetidos os povoamentos, indica que estas espécies são oportunistas quando comparadas com outras, apresentando grande capacidade de colonizar habitats alterados pelo homem e com baixa complexidade estrutural.

Das 13 espécies do gênero Acromyrmex Mayr 1865 registradas para o Rio Grande do Sul (MAYHÉ-NUNES \& DiEHLFLEIG 1994) foram coletadas três nas áreas avaliadas em Capivari do Sul: A. ambiguus Emery 1888, A. laticeps Emery 1905 e A. lundi (Guérin 1838). As formigas do gênero Acromyrmex destacam-se por cortarem um grande número de diferentes vegetais e pelos elevados danos que causam especialmente nas florestas comerciais (Fowler et al. 1986). Segundo Hölldobler \& WiLSON (1990), este gênero juntamente com Atta Fabricius, 1804 são considerados como os herbívoros dominantes da Região Neotropical consumindo muito mais vegetação do que qualquer outro grupo com diversidade taxonômica comparável. Também pertencentes à tribo Attini, foram coletados representantes dos gêneros Apterostigma Mayr 1865, Cyphomyrmex Mayr 1862 e Trachymyrmex (Forel, 1893). Cabe destacar que embora as espécies dos dois primeiros gêneros sejam fungicultoras, não utilizam matéria vegetal viva para cultivar o fungo simbionte, enquanto que as colônias de Trachymyrmex, mesmo utilizando material vegetal vivo, por serem bastante pequenas, não causam danos à produção vegetal.

Os resultados obtidos podem ser considerados um passo inicial para o conhecimento da formicifauna em povoamentos de eucalipto implantados em restingas no Rio Grande do Sul. No entanto, sugerem-se estudos comparativos sobre a ecologia das comunidades de formigas em florestas implantadas e em remanescentes naturais do ecossistema de restingas, com a finalidade de se compreender os fatores responsáveis pelos padrões de distribuição destes insetos.
Este conhecimento poderá fornecer subsídios valiosos aos planos de utilização e preservação das restingas, bem como aos programas de manejo de florestas implantadas neste ecossistema.

Agradecimentos. À FLOSUL - Florestamento do Sul Ltda., por ter financiado e permitido o desenvolvimento deste trabalho em suas propriedades, à CAPES pela concessão da bolsa de mestrado ao primeiro autor, aos bolsistas Laura V.A. Menzel, Fernando A. Schmidt e Marta Bencke pelo auxílio nas coletas e ao Dr. J.H.C. Delabie do Laboratório de Mirmecologia, CEPEC/CEPLAC (Itabuna, BA) pela identificação de algumas espécies.

\section{REFERÊNCIAS}

Andersen, A. N. 1991. Responses of ground-foraging ant communities to three experimental fire regimes in a savanna forest of tropical Australia. Biotropica 23: 575-585.

ANDERSEN, A. N. 1997. Using ants as bioindicators: multiscale issues in ant community ecology. Ecology 1: 8. <http://www.consecol.org/ vol1/iss $1 /$ ant. $8>$.

Bolton, B. 1994. Identification guide to the ant genera of the world. Cambridge, Harvard University Press, 222 p.

Bolton, B. 1995. A new general catalogue of the ants of the world. Cambridge, Harvard University Press, 504 p.

BonNET, A. \& B. C. Lopes. 1993. Formigas de dunas e restingas da praia da Joaquina, Ilha de Santa Catarina, SC (Insecta: Hymenoptera). Biotemas 6(1): 107-114.

Caldas, A. \& P. R. S. Moutinho. 1993. Composição e diversidade da fauna de formigas (Hymenoptera, Formicidae) em áreas sob remoção experimental de árvores na Reserva Florestal de Linhares, ES, Brasil. Revista Brasileira de Entomologia 37(2): 299-305.

Colwell, R. K. 1997. Estimates: estatistical estimation of species richness and shared species from samples. Version 5.0.1. <http:// viceroy.eeb.uconn.edu/ estimate>.

Dienl-Fleig, E. 1997. Ocorrência de Acromyrmex em áreas com distintos níveis de perturbação antrópica no Rio Grande do Sul. Acta Biologica Leopoldensia 19(2): 165-171.

Diehl-Fleig, E.; Ed. Diehl-Fleig \& C. E. D. Sanhudo. 2000. Mirmecofauna de solo nas dunas da Praia Grande e no Morro da Guarita no Município de Torres, RS, Brasil. Acta Biologica Leopoldensia 22(1): 37 43.

Flores, D. G.; C. L. Goettert \& E. Diehl. 2002. Comunidades de formigas em Inga marginata (Fabaceae) e Jacaranda micrantha (Bignoniaceae) em área suburbana. Acta Biologica Leopoldensia 24(2): 147-156.

FowleR, H. G. 1995. Diversity estimates: Ant communities and the rare ant species (Hymenoptera: Formicidae) in fauna of a sub-tropical island. Revista de Matemática e Estatística 13: 29-38.

Fowler, H. G.; V. Pereira-da-Silva \& N. B. Saes. 1986. Economics of grass-cutting ants, p. 18-35. In: C. S. LofGren \& R. K. VANDer Meer (eds.) Fire ants and leaf-cutting ants: biology and management. Boulder, Westview Press, $435 \mathrm{p}$.

Fowler, H. G.; L. C. Forti; C. R. F. Brandẽo; J. H. C. Delabie \& H. L. VASCONCELOS. 1991. Ecologia nutricional de formigas, p. 131-223. In: A. P. Panizzi \& J. R. P. Parra (eds.) Ecologia nutricional de insetos e suas implicações no manejo de pragas. São Paulo, Manole, $359 \mathrm{p}$.

Grürzmacher, D. D.; A. E. Loeck \& A. H. Medeiros. 2002. Ocorrência de formigas cortadeiras na Região da Depressão Central do estado do Rio Grande do Sul. Ciência Rural 32(2): 185-190.

Gusmão, L. \& A. E. Loeck. 1999. Distribuição geográfica de formigas cortadeiras do gênero Acromyrmex (Hymenoptera: Formicidae) na Zona Sul do estado do Rio Grande do Sul. Revista Brasileira de Agrociência 5(1): 64-67.

Haubert, F.; E. Diehl-Fleig \& A. J. Mayhé-Nunes. 1998. Mirmecofauna de 
solo do município de São Leopoldo, RS: Levantamento preliminar. Acta Biologica Leopoldensia 20(1): 103-108.

Hölldobler, B. \& E. O. Wilson. 1990. The Ants. Cambridge, The Belknap Press of Harvard University, $732 \mathrm{p}$.

Junqueira, L. K.; E. Diehl \& Ed. Diehl-Fleig. 2001. Formigas (Hymenoptera: Formicidae) Visitantes de Ilex paraguariensis (Aquifoliaceae). Neotropical Entomology 30(1): 161-164.

KempF, W. W. 1972. Catálogo abreviado das formigas da Região Neotropical (Hym., Formicidae). Studia Entomologica 15(1-4): $1-134$.

LEAL, I. R. \& B. C. Lopes. 1992. Estrutura das comunidades de formigas (Hymenoptera: Formicidae) de solo e vegetação no Morro da Lagoa da Conceição, Ilha de Santa Catarina, SC. Biotemas 5(1): 107122.

Lobry DE Bruyn, L. A. 1999. Ants as bioindicators of soil function in rural environments. Agriculture, Ecosystems and Environment 74: $425-441$

Lopes, B. C. \& I. R. LeAL. 1991. Levantamento preliminar de formigas (Hymenoptera: Formicidae) de solo e vegetação em um trecho de Mata Atlântica, Morro da Conceição, Ilha de Santa Catarina, SC. Biotemas 4(2): 51-59.

MAJER, J. D. 1983. Ants: bio-Indicators of minesite rehabilitation, landuse, and land conservation. Environment Management 7(4): 375-383.

MAJER, J. D. 1996. Ant recolonization of rehabilitated bauxite mines at Trombetas, Pará, Brazil. Journal of Tropical Ecology 12: 257273.

Majer, J. D. \& O. G. Nichols. 1998. Long-term recolonization patterns of ants in Westerm Australian rehabilitated bauxite mines with reference to their use as indicators of restoration success. Journal of Applied Ecology 35: 161-182.

Marinho, C. G. S.; R. Zanetti; J. H. C. Delabie; M. N. Schlindwein. \& L. S. Ramos. 2002. Diversidade de formigas (Hymenoptera: Formicidae) da serapilheira em eucaliptais (Myrtaceae) e área de cerrado de Minas Gerais. Neotropical Entomology 31(2): 187-195.

Matos, J. Z.; C. N. Yamanaka; T. T. Castellani; B. C. Lopes. 1994. Comparação da fauna de formigas de solo em áreas de plantio de Pinus elliottii, com diferentes graus de complexidade estrutural (Florianópolis, SC). Biotemas 7(1-2): 57-64.

Mayhé-Nunes, A. J. \& E. Diehl-Fleig. 1994. Distribuição de Acromyrmex
(Hymenoptera: Formicidae) no Rio Grande do Sul. Acta Biologica Leopoldensia 16(1): 115-118.

McGeoch, M. A. \& S. L. Chown. 1998. Scaling up the value of bioindicators. TREE 13(2): 46-47.

NEw, T. R. 1996. Taxonomic foxus and quality control in insect surveys for biodiversity conservation. Australian Journal of Entomology 35: $97-106$.

Oliveira, M. A.; T. M. C. Della Lucia; A. P. Araújo \& A. P. Da Cruz. 1995. A fauna de formigas em povoamentos de eucalipto e mata nativa no estado do Amapá. Acta Amazonica 25(1-2): 117-126.

Poggiani, F. \& R. E. Oliveira. 1998. Indicadores para conservação dos núcleos de vida silvestre. Série Técnica IPEF 12(31): 45-52.

Romero, H. \& K. JAFFÉ. 1989. A comparison of methods for sampling ants (Hymenoptera, Formicidae) in savannas. Biotropica 21: 348 325.

SAnhudo, C. E. \& E. Diehl-Fleig. 1999. Espécies de formigas cortadeiras e densidade de ninhos em áreas de reflorestamento. Naturalia 24 (n.esp): 123-124.

Santos, J. C. Dos \& E. Dienl. 2001. Comunidades de formigas em Acacia mearnsii (Mimosaceae) e Baccharis dracunculifolia (Asteraceae). Acta Biologica Leopoldensia 23(2): 181-190.

SiLVA, R. R. DA \& C. R. F. BRANDÃo. 1999. Formigas (Hymenoptera: Formicidae) como indicadoras da qualidade ambiental e da biodiversidade de outros invertebrados terrestres. Biotemas 12(2): $55-73$.

Tavares, A. A.; P. C. Bispo \& A. C. S. Zanzini. 2001. Comunidades de formigas epigéicas (Hymenoptera, Formicidae) em áreas de Eucalyptus cloeziana F. Muell e de vegetação nativa numa região de cerrado. Revista Brasileira de Entomologia 45(3): 251256.

VAsconcelos, H. L. 1998. Respostas das formigas à fragmentação florestal. Série Técnica IPEF 12(32): 95-98.

VianA, V. M. 1995. Conservação da biodiversidade de fragmentos de florestas tropicais em paisagens intensamente cultivadas, p. 135154. In: Abordagens interdisciplinares para a conservação da biodiversidade e dinâmica do uso da terra no Novo Mundo. Gainesville, Conservation International do Brasil/ UFMG/ University of Florida.

Wilson, E. O. 1976. Which are the most prevalent ant genera? Studia Entomologica 19(1-4): 187-200.

Recebido em 12.VIII.2003; aceito em 15.I.2004 